\title{
TRENDS IN USE OF SURGICAL MESH FOR PELVIC ORGAN PROLAPSE
}

\author{
Michele JONSSON FUNK, PhD ${ }^{1,2}$, Autumn L. EDENFIELD, MD ${ }^{3}$, Ms. Virginia PATE, MS ${ }^{1}$, \\ Anthony G. VISCO, MD $^{3}$, Alison C. WEIDNER, $\mathbf{M D}^{3}$, and Jennifer M. WU, MD, $\mathbf{M P H}^{3}$ \\ ${ }^{1}$ Department of Epidemiology, Gillings School of Global Public Health, University of North \\ Carolina at Chapel Hill, Chapel Hill, NC \\ ${ }^{2}$ Center for Women's Health Research, University of North Carolina at Chapel Hill, Chapel Hill, \\ $\mathrm{NC}$ \\ ${ }^{3}$ Division of Urogynecology, Department of Obstetrics and Gynecology, Duke University, Durham, \\ $\mathrm{NC}$
}

\begin{abstract}
Objective-Limited data exist on the rates of pelvic organ prolapse procedures utilizing mesh. The objective of this study was to examine trends in vaginal mesh prolapse procedures (VM), abdominal sacrocolpopexy (ASC) and minimally-invasive sacrocolpopexy (MISC) from 20052010 .
\end{abstract}

Study Design-We utilized de-identified, adjudicated healthcare claims data from across the United States from 2005-2010. Among women $\geq 18$ years, we identified all mesh prolapse procedures based on CPT codes (57267 for VM, 57280 for ASC and 57425 for MISC). VM procedures included all vaginal prolapse surgeries in which mesh was placed, whether in the anterior, apical or posterior compartment. We estimated rates per 100,000 person-years (100,000py) and 95\% confidence intervals (95\% CI).

Results-During 78.5 million person-years of observation, we identified 60,152 mesh prolapse procedures, for a rate of 76.0 per 100,000py 100,000py (95\% CI: 73.6, 78.5). Overall, VM comprised $74.9 \%$ of these surgeries for an overall rate of 56.9 per 100,000py (95\% CI: 55.0, 58.9). Rates of ASC and MISC were considerably lower at 12.0 per 100,000py (95\% CI: 11.6, 12.5) and 9.5 per 100,000py (95\% CI: 9.2, 9.9), respectively. Among sacrocolpopexies, ASC was more common than MISC in 2005-2007; however, since 2007, the rate of MISC has increased while the rate of ASC has decreased. Regarding trends by age, VM was considerably more common than sacrocolpopexies at all ages, and ASC was more common than MISC in women over 50 years.

\footnotetext{
() 2012 Mosby, Inc. All rights reserved.

Corresponding Author: Jennifer M. Wu, MD, MPH, Duke University Medical Center, Box 3192, 5324 McFarland Avenue, Suite 310 , Durham, North Carolina 27707, Work: (919) 401-1006, Cell: (919) 452-4577, Fax: (919) 401-1033, jennifer.wu@ duke.edu. Disclosure: None of the authors have a conflict of interest

The content is solely the responsibility of the authors and does not necessarily represent the official views of the Agency for Healthcare Research and Quality.

Reprints will not be available

Accepted as oral poster presentation at the 33rd Annual Meeting of the American Urogynecologic Society, Chicago, IL, October 3-6, 2012.

Publisher's Disclaimer: This is a PDF file of an unedited manuscript that has been accepted for publication. As a service to our customers we are providing this early version of the manuscript. The manuscript will undergo copyediting, typesetting, and review of the resulting proof before it is published in its final citable form. Please note that during the production process errors may be discovered which could affect the content, and all legal disclaimers that apply to the journal pertain.
} 
Conclusions-From 2005 to 2010, the rate of mesh prolapse procedures has increased, with vaginal mesh surgeries constituting the vast majority.

\section{Keywords}

pelvic organ prolapse; sacrocolpopexy; surgery; transvaginal mesh; trends

\section{INTRODUCTION}

The recent FDA safety communication regarding vaginal mesh for pelvic organ prolapse has drawn attention to the surgical management of this highly prevalent condition. ${ }^{1}$ In the July 2011 update on the original 2008 public health notification ${ }^{2}$, the FDA stated that "based on an updated analysis of adverse events reported to the FDA and complications described in the scientific literature, the FDA identified surgical mesh for transvaginal repair of POP as an area of continuing serious concern."1 The American Congress of Obstetricians and Gynecologists (ACOG) and the American Urogynecologic Society (AUGS) have recognized the critical importance of complications such as mesh exposure, erosion and contracture, and the symptoms associated with these complications. ${ }^{3}$

The first mesh "kits" to aid in the insertion of vaginal synthetic graft material for prolapse were cleared by the FDA in 2004 and marketed by American Medical Systems (AMS) under the names Apogee ${ }^{\mathrm{TM}}$ and Perigee ${ }^{\mathrm{TM}}$ Systems. ${ }^{4,5}$ Since then, there has been an influx of new prolapse mesh devices introduced in the United States. ${ }^{6}$ Sales data from manufacturers indicate that 300,000 women underwent pelvic organ prolapse surgery in $2010 .{ }^{6}$ Of these, one-third utilized mesh $(\sim 100,000)$, and three-quarters of these mesh procedures were transvaginal surgeries. Thus, approximately, 75,000 procedures involved transvaginal placement of mesh. Despite these estimates, population-based estimates of vaginal and abdominal mesh prolapse surgeries are extremely limited. Furthermore, utilization of mesh for prolapse repair categorized by age and region is lacking.

Several studies have evaluated national trends in prolapse surgeries overall ${ }^{7-10}$, but these estimates provide limited information regarding mesh prolapse procedures specifically. One reason is that International Classification of Diseases, $9^{\text {th }}$ Revision, Clinical Modification (ICD-9-CM) codes for prolapse graft materials (i.e. codes 70.53, 70.54, 70.55, 70.94 and 70.95) were not introduced until 2007. ${ }^{11}$ Another limitation is that ICD-9-CM procedure codes, which are utilized by a majority of national databases, do not incorporate the more specific Current Procedural Terminology (CPT) codes. Because of these limitations, the rates of specific mesh procedures have not been reported, and the impact on rates of transvaginal mesh procedures after the two recent FDA notifications in 2008 and 2011 is relatively unknown. It is critical to understand whether the FDA safety notifications have translated into changes in the rates of transvaginal mesh procedures.

Given the knowledge gaps in the existing literature, we sought to use the more informative CPT codes in a population-based analysis to estimate trends in prolapse mesh procedures from $2005-2010$. Furthermore, we wanted to estimate the rates of specific prolapse procedures, such as vaginal mesh procedures (VM), abdominal sacral colopopexy (ASC) and minimally-invasive sacrocolpopexy (MISC) and to estimate the trends in these surgeries based on age and region of the U.S. 


\section{MATERIALS AND METHODS}

\section{Data Source}

This analysis is based on Thomson Reuters' MarketScan ${ }^{\circledR}$ Commercial Claims and Encounters $(C C \& E)$ database and Medicare Supplemental and Coordination of Benefits database from 2005 - 2010 (copyright $(9) 2011$ Thomson Healthcare Incorporated Inc. All rights reserved). ${ }^{12}$ These data represent the medical encounters of individuals with employment-based insurance including spouses, dependents, and retirees. Specifically, the databases contain de-identified, individual-level inpatient, outpatient, and pharmacy claims as well as detailed enrollment data from approximately 100 payers in the United States. Claims and enrollment data are validated to ensure completeness, accuracy, and reliability. Although the data are de-identified, unique individuals can be followed over time using encrypted identification numbers. The databases contain adjudicated, paid claims for approximately 28.3 million individuals in 2005 , increasing to 48.8 million in 2010 . Of note, in $2010,55.3 \%$ of the U.S. population had employment-based insurance. ${ }^{13}$ This study was determined to be exempt from further review by the Institutional Review Board at the University of North Carolina at Chapel Hill.

\section{Pelvic Organ Prolapse Mesh Surgery Rates}

The study population included all women aged 18 years or older from $2005-2010$. We utilized CPT codes to identify any prolapse procedures involving mesh placement. A vaginal mesh procedure was defined by the CPT code 57267 (insertion of mesh or other prosthesis for repair of pelvic floor defect, each site, vaginal approach). Because this code is listed in addition to CPT code(s) for the primary procedure(s), we defined a vaginal mesh procedure if CPT 57267 was listed at least once on a specific surgery date. For example, if mesh was placed in both the anterior and posterior compartment and CPT 57267 was listed twice, this surgery would be counted as a single VM procedure. We chose to evaluate trends starting in 2005 because the CPT code 57267 was released on January 1, 2005. ${ }^{14}$ We opted to use CPT codes instead of ICD-9-CM codes, as ICD-9-CM vaginal mesh codes were not introduced until 2007. ${ }^{11}$ For sacrocolpopexies, ASC were identified using CPT 57280, which indicates "colpopexy, abdominal approach." Minimally-invasive sacrocolpopexies, MISC, were defined by CPT 57425, a code that was released in 2004. MISC represents both laparoscopic and robotic-assisted laparoscopic sacrocolpopexies as CPT codes distinguishing these two types of surgeries do not exist.

Rates were calculated by dividing the total number of surgeries in each category (VM, ASC and MISC) by the total person-time at risk. In order to calculate person-time at risk, we summed all periods of time when eligible women were enrolled in a health plan that contributed to the database.

We also evaluated common concurrent procedures with VM, ASC and MISC. Specifically, we evaluated concomitant sling, CPT 57288, as well as concomitant hysterectomy. We evaluated the type of hysterectomy (total versus supracervical) based on CPT codes. Total hysterectomy included total abdominal (CPT 58150, 58152, 58200, 58210), total vaginal (CPT 58260, 58262, 58263, 58267, 58270, 58275, 58280, 58285, 58290, 58291, 58292, $58293,58294)$, and total laparoscopic which also included laparoscopic assisted vaginal hysterectomy (CPT 58545, 58546, 58548, 58550, 58552, 58553, 58554, 58570, 58571, 58572,58573 ). Supracervical hysterectomies included both abdominal supracervical (CPT 58180) and laparoscopic supracervical (CPT 58541, 58542, 58543, 58544). 


\section{Statistical Analysis}

We estimated the rate of any prolapse mesh surgery overall, as well as procedure-specific rates for VM, ASC and MISC. In addition, we estimated the procedure and calendar-year, procedure and location (inpatient vs outpatient), procedure and age, and procedure and region specific rates for prolapse mesh surgery. We estimated $95 \%$ confidence intervals (CIs) based on the Poisson distribution. Rates and CIs are reported per 100,000 person-years (100,000py).

In order to adjust for differences in the age distribution over calendar time and across regions, we estimated region-specific and calendar-year specific rates using Poisson regression while adjusting for age using 15 categorical variables $(18-24,25-29,30-34,35-$ $39,40-44,45-49,50-54,55-59,60-64,65-69,70-74,75-79,80-84,85-89,90+)$. Wald chi-square statistics were used to test the significance of independent predictors with a twosided alpha $=0.05$. All confidence intervals were estimated and statistical tests were conducted using Poisson regression with the dispersion parameter empirically estimated by the Pearson chi-square divided by the degrees of freedom to account for overdispersion. Analyses were performed using SAS, v9.2 (SAS Institute, Cary, NC).

\section{RESULTS}

From 2005 - 2010, women aged 18 years and older contributed a total of 78,496,836 person-years. Among these women, there were 60,152 pelvic organ prolapse procedures which involved the use of mesh. Table 1 lists the person-time contribution for the population at-risk, divided by calendar year, age group (18-29, 30-39, 40-49, 50-59, 60-69, 70-79, $80+$ years), and region of the U.S. Furthermore, Table 1 describes the overall number of prolapse procedures with mesh, as well as the specific type of surgery, whether VM, ASC or MISC.

The overall rate of any prolapse surgery with mesh was 76.0 per 100,000py (95\% CI 73.6, 78.5) (Table 2). VM comprised $74.9 \%$ of these surgeries for an overall rate of 56.9 per 100,000py (95\% CI: 55.0, 58.9). Rates of ASC and MISC were considerably lower at 12.0 per 100,000py (95\% CI: 11.6, 12.5) and 9.5 per 100,000py (95\% CI: 9.2, 9.9), respectively (Table 2). While approximately half of the VM and MISC procedures were performed on an outpatient basis, a majority of the ASC procedures were inpatient surgeries (Table 2).

Regarding the trend for the total number of prolapse mesh procedures from 2005-2010, there was a significant increase from 53.5 per 100,000py $(95 \%$ CI 49.4, 57.9) in 2005 to 83.7 per 100,000py (95\% CI 77.3, 90.5) in 2010 (p<.0001) (Figure 1). There was also a significant increase for VM procedures from 36.7 to 60.8 per 100,000py ( $\mathrm{p}<.0001$ ) (Table 2; Figure 1). For VM, the rate appears to increase from 2005 to 2008 and then level off from 2008 to 2010. Among sacrocolpopexies, the rate of ASC significantly decreased from 14.8 per 100,000py (95\% CI 13.5, 16.2) in 2005 to 9.9 per 100,000py (95\% CI 9.1, 10.8) in 2010 $(\mathrm{p}<.0001)$. In contrast, the rate of MISC significantly increased from 4.3 to 15.9 per 100,000py (p<.0001). By 2010, the rate of MISC was higher than that of ASC (Table 2).

VM was the most common type of prolapse mesh surgery with age-specific rates higher than ASC and MISC for all age cohorts (Table 2, Figure 2). The age-specific rate for VM was highest in women aged 70-79 years, while the rates of ASC and MISC were highest in women aged 60-69 years (Table 2).

When evaluating trends by region, Figure 3 depicts the age-specific rates for any prolapse mesh procedure from $2005-2010$ by region of the U.S. This figure illustrates that all regions had significantly higher rates of prolapse mesh surgeries compared to the Northeast, after 
adjusting for age $(\mathrm{p}<.0001)$. Furthermore, the rates were highest in the South compared to the other regions of the U.S (Table 2).

We also wanted to describe common concurrent procedures that occurred with the use of mesh for prolapse. A concomitant sling occurred in 39.9\% $(n=24,024)$ and a concurrent hysterectomy in $27.2 \%(n=16,363)$ of mesh prolapse procedures. Among hysterectomies, $14.6 \%(\mathrm{n}=2,385)$ were supracervical.

\section{COMMENT}

In this population-based analysis of more than 60,000 pelvic organ prolapse procedures utilizing mesh, vaginal mesh represented the majority (74.9\%) of surgeries. From 2005 to 2010 , there has been a significant increase in prolapse mesh procedures, largely due to the increase in VM, since rates of sacrocolpopexies (both ASC and MISC) remained relatively low. Interestingly, the rate of ASC declined over this time period while the rate of MISC increased. As for trends based on age, $\mathrm{VM}$ was the most common procedure across all age groups. Despite the initial FDA public health notification in October $2008,{ }^{2}$ we did not see evidence of a dramatic decrease in the rate of VM procedures after 2008 but this rate did appear to level off from $2008-2010$.

Our CPT-based approach to describe mesh use during prolapse surgery is novel, as prior literature is limited. In 2010, the FDA estimated that of the 300,000 prolapse surgeries performed annually, one-third utilized mesh grafts. ${ }^{6}$ Our finding that $74.9 \%$ of the mesh procedures were VM mirrors industry estimates that three-quarters of mesh procedures are transvaginal. These data confirm the popularity of these surgeries in current practice.

The high rate of VM procedures further emphasizes the importance of potentially serious complications of these procedures. The FDA July 2011 update stated that there were 1,503 reported complications associated with surgical mesh devices for prolapse from January 1 , 2008 through December 31, 2010. ${ }^{1}$ The most common complications included "mesh erosion through the vagina (also called exposure, extrusion or protrusion), pain, infection, bleeding, pain during sexual intercourse (dyspareunia), organ perforation, and urinary problems. There were also reports of recurrent prolapse, neuro-muscular problems, vaginal scarring/shrinkage, and emotional problems". ${ }^{1}$ Unfortunately, many of these complications required additional interventions, and anecdotally, many urogynecologists are witnessing a dramatic increase in the number of patients with mesh complications in their practices. While the existing literature suggests that there may be a role for mesh augmentation in the anterior compartment for improved anatomic success $3,6,15,16$, the FDA stated that it is not clear that that mesh augmentation is more effective than compared to native tissue repairs. ${ }^{1,6}$ In their joint committee opinion, ACOG and AUGS recommended that mesh augmentation be reserved for high-risk individuals in whom the benefit of mesh placement outweighs the potential risks. ${ }^{3}$ While the initial FDA notification in 2008 did not have a dramatic impact on VM rates, it will be important to monitor the impact of the July 2011 update on rates of VM procedures in the coming years.

In addition to an increase in VM procedures from 2005 to 2010, our data shows an increase in the MISC rate and a decrease in ASC rate over the study period. Because of the limited detail of CPT codes in current use, we are unable to distinguish between laparoscopic and robotic-assisted sacrocolpopexies, and we cannot definitively state what proportion of MISC is due to each of these approaches. Jones et al. ${ }^{9}$ showed that laparoscopic prolapse surgeries increased among inpatients from 1979 to 2006, and Bradley et al. ${ }^{10}$ reported that minimallyinvasive inpatient prolapse procedures increased from $4.8 \%$ in 1998 to $9.4 \%$ in 2007 . While the laparoscopic sacrocolpopexy was first described in $1994{ }^{17}$, the robotic-assisted approached was first reported in $2004 .{ }^{18,19}$ In 2005, the da Vinci Surgical System (Intuitive 
Surgical, Inc., Sunnyvale, CA) was approved by the FDA for use in gynecology. ${ }^{20}$ Given this timeline, we postulate the greater proportion of the increase in the MISC rate may be secondary to an increase in the number of robotic-assisted sacrocolpopexies.

In our study, we found that a total hysterectomy (TAH) was more common than a supracervical hysterectomy ( $\mathrm{SCH}$ ) for both ASC and MISC. The literature is conflicting regarding the relationship of type of hysterectomy and the risk of mesh erosion.(22) Several studies suggest that there may be no difference between the risk of mesh erosion after ASC between TAH and $\mathrm{SCH}^{21,22}$ However, a large randomized multi-centered study found an increased risk of mesh or suture erosion with ASC with concomitant TAH (14\%) compared to those with a prior hysterectomy (4\%). ${ }^{23}$ Furthermore, two studies which assessed roboticassisted MISC found no erosions among subjects who had concomitant $\mathrm{SCH} .{ }^{24,25}$ These data suggest concurrent $\mathrm{SCH}$ may decrease the risk of mesh erosion following a sacrocolpopexy. However, minimally invasive $\mathrm{SCH}$ is not without its trade-offs, as there is the need for continued cervical cancer screening and the risk of unanticipated premalignant or malignant uterine pathology in a morcellated SCH specimen, ${ }^{25-27}$ Further research and education regarding hysterectomy choice and ASC and MISC is warranted.

The largest proportion of mesh prolapse surgeries in our study was performed in women aged 60-69 years. This age range is similar to that of prior studies investigating inpatient prolapse surgery trends in the U.S, as most procedures were performed in women aged 6079 years. ${ }^{10,28}$ Prior investigations have also shown the mean age of women undergoing both inpatient and outpatient prolapse surgery in the U.S. has increased over the last decade. ${ }^{8,9}$ Regional trends have consistently shown that the South has the highest rates of prolapse surgery, though this is largely unexplained. ${ }^{9}, 10,28$ This trend could potentially be related several factors: the increased tendency of physicians in the South to perform surgery for prolapse, a higher prevalence of disease in the South, or greater preference of surgery as a treatment option for prolapse among Southern women. These factors have not been thoroughly investigated or well explored.

In this study, we present data regarding the rates of prolapse procedures which involve the use of mesh. The strengths of this study include the use of a population-based database of adjudicated healthcare claims, which provides an exceptionally large cohort to evaluate. Furthermore, the utilization of CPT versus ICD-9 codes allowed us to evaluate specific prolapse procedures - i.e. VM vs ASC vs MISC, as well as the whether these procedures were performed on an inpatient or outpatient basis. Lastly, we evaluated trends starting in 2005 , as the CPT vaginal mesh codes were introduced that year. Evaluation of prolapse mesh procedures prior to 2005 may not be particularly accurate as specific codes were not available.

Regarding limitations, it is possible that some VM procedures were not appropriately coded in 2005, as CPT code 57267 was introduced that year; however, the significant upward trend in VM rates suggests that there has likely been an increase in the use of these procedures. Another factor is that we did not have details regarding the surgical procedure in terms of the type of mesh utilized as well as the method in which mesh was placed. For MISC, the CPT code 57425 for laparoscopic colpopexy could also represent laparoscopic uterosacral ligament suspensions; thus it is possible that we may have slightly overestimated the rate of sacral colpopexies utilizing mesh. In addition, we were unable to evaluate certain demographic or clinical factors, such race, body mass index (BMI), indication for surgery (i.e. primary prolapse surgery or surgery for recurrent prolapse), or stage of prolapse. Lastly, our database included only those with employer-based insurance, and thus our results may not be generalizable to those without other types of insurance or self-pay patients. Fortunately, with the Medicare supplemental database, we were able to evaluate older 
women who had employer-based insurance and then transitioned into Medicare at 65 years of age.

In conclusion, transvaginal mesh procedures comprised $75 \%$ of all prolapse mesh surgeries, and VM was the most common mesh procedure across all age groups. Although rates of vaginal mesh procedures were significantly higher than abdominal and minimally-invasive sacrocolpopexies, the rate of MISC increased while that of the ASC decreased from 2005 to 2010. Although we did not see a dramatic decrease in the rate of VM procedures after the initial FDA public health notification in 2008, the rate of VM appeared to level off from 2008-2010. It will be important to evaluate the impact of the July 2011 FDA notification, as this statement highlighted serious complications after transvaginal mesh prolapse surgery more emphatically than the 2008 statement. Beyond evaluating trends in surgery rates, future studies should strive for rigorous, comparative effectiveness studies that incorporate patient-centered outcome measures as well as long-term, population-based data regarding complications of vaginal mesh. Despite the popularity of VM procedures, population-based data regarding the rates of repeat surgery to manage complications such as mesh erosion/ exposure and mesh contracture are strikingly lacking.

\section{Acknowledgments}

This project was supported by grant number K02HS017950 from the Agency for Healthcare Research and Quality (AHRQ) (M.J.F) and grant number K23HD068404 from the Eunice Kennedy Shriver National Institute of Child Health \& Human Development (J.M.W).

\section{References}

1. [Accessed on June 12, 2012] FDA Safety Communication: Update on serious complications associated with transvaginal placement of surgical mesh for pelvic organ prolapse. Issued on July 13, 2011at http://www.fda.gov/medicaldevices/safety/alertsandnotices/ucm262435.htm

2. [Accessed on June 12, 2012] FDA Public Health Notification: Serious Complications Associated with Transvaginal Placement of Surgical Mesh in Repair of Pelvic Organ Prolapse. Issued: October 20, 2008at http://www.fda.gov/medicaldevices/safety/alertsandnotices/publichealthnotifications/ ucm061976.htm

3. American College of Obstetricians and Gynecologists and American Urogynecology Society. Committee Opinion no 513: vaginal placement of synthetic mesh for pelvic organ prolapse. Obstet Gynecol. 2011; 118:1459-64. [PubMed: 22105294]

4. FDA 510(k) Premarket Notification. AMS APOGEE VAULT SUSPENSION SYSTEM; Number K040537at http://www.accessdata.fda.gov/scripts/cdrh/cfdocs/cfPMN/pmn.cfm?ID=14536

5. FDA 510(k) Premarket Notification. AMS PERIGEE SYSTEM; Number K040623at http:// www.accessdata.fda.gov/scripts/cdrh/cfdocs/cfPMN/pmn.cfm?ID=14611

6. [Accessed on June 12, 2012] FDA Executive Summary: Surgical mesh for the treatment of women with pelvic organ prolapse and stress urinary incontinence. Sep 8-9. 2011 at http://www.fda.gov/ downloads/AdvisoryCommittees/CommitteesMeetingMaterials/MedicalDevices/ MedicalDevicesAdvisoryCommittee/ObstetricsandGynecologyDevices/UCM270402.pdf

7. BOYLES SH, WEBER AM, MEYN L. Procedures for pelvic organ prolapse in the United States, 1979-1997. Am J Obstet Gynecol. 2003; 188:108-15. [PubMed: 12548203]

8. EREKSON EA, LOPES VV, RAKER CA, SUNG VW. Ambulatory procedures for female pelvic floor disorders in the United States. Am J Obstet Gynecol. 2010

9. JONES KA, SHEPHERD JP, OLIPHANT SS, WANG L, BUNKER CH, LOWDER JL. Trends in inpatient prolapse procedures in the United States, 1979-2006. Am J Obstet Gynecol. 2010; 202:501, e1-7. [PubMed: 20223444]

10. BRADLEY SL, WEIDNER AC, SIDDIQUI NY, GANDHI MP, WU JM. Shifts in national rates of inpatient prolapse surgery emphasize current coding inadequacies. Female Pelvic Med Reconstr Surg. 2011; 17:204-8. [PubMed: 22453854] 
11. Centers for Disease Control and Prevention. Classification of Diseases, Conversion Table of New ICD-9-CM Codes. Oct. 2011 Available at http://www.cdc.gov/nchs/data/icd9/CNVTB12.pdf. Retrieved June 26, 2012

12. Thomson Reuters MarketScan ${ }^{\circledR}$ Commercial Claims and Encounters Database. [Accessed on June 12, 2012] Source: RED BOOK® and MarketScan ${ }^{\circledR}$ are registered trademarks of Thomson Reuters (Healthcare) Inc. 2000-2009. at http://thomsonreuters.com/products_services/healthcare/ healthcare_products/pharmaceuticals/mktscan_res_db/

13. DENAVAS-WALT, C.; PROCTOR, BD.; SMITH, JC. US Census Bureau, Current Population Reports, P60-239, Income, Poverty, and Health Insurance Coverage in the United States: 2010. Washington, D.C: U.S. Government Printing Office; 2011. at http://www.census.gov/prod/ 2011pubs/p60-239.pdf

14. Center for Medicare and Medicaid Services. January 2005 Update to the Medicare Non-OPPS Outpatient Code Editor (OCE) Specifications Version 20.1 for Bills from Hospitals Not Paid Under the Outpatient Prospective Payment Systems (OPPS), MLN Matters Number: MM3621. Available at https://www.cms.gov/Outreach-and-Education/Medicare-Learning-Network-MLN/ MLNMattersArticles/Downloads/MM3621.pdf. Retrieved June 26, 2012

15. NIEMINEN K, HILTUNEN R, TAKALA T, et al. Outcomes after anterior vaginal wall repair with mesh: a randomized, controlled trial with a 3 year follow-up. Am J Obstet Gynecol. 2010; 203:235, e1-8. [PubMed: 20494332]

16. ALTMAN D, VAYRYNEN T, ENGH ME, AXELSEN S, FALCONER C. Short-term outcome after transvaginal mesh repair of pelvic organ prolapse. Int Urogynecol J Pelvic Floor Dysfunct. 2007

17. NEZHAT CH, NEZHAT F, NEZHAT C. Laparoscopic sacral colpopexy for vaginal vault prolapse. Obstet Gynecol. 1994; 84:885-8. [PubMed: 7936532]

18. ELLIOTT DS, FRANK I, DIMARCO DS, CHOW GK. Gynecologic use of robotically assisted laparoscopy: Sacrocolpopexy for the treatment of high-grade vaginal vault prolapse. Am J Surg. 2004; 188:52S-56S. [PubMed: 15476652]

19. DI MARCO DS, CHOW GK, GETTMAN MT, ELLIOTT DS. Robotic-assisted laparoscopic sacrocolpopexy for treatment of vaginal vault prolapse. Urology. 2004; 63:373-6. [PubMed: 14972496]

20. FDA 510(k) Premarket Notification. INTUITIVE SURGICAL DA VINCI SURGICAL SYSTEM AND ENDOSCOPIC INSTRUMENTS; Number K050404Available at http:// www.accessdata.fda.gov/scripts/cdrh/cfdocs/cfPMN/pmn.cfm?ID=17591. Retrieved June 26, 2012

21. WU JM, WELLS EC, HUNDLEY AF, CONNOLLY A, WILLIAMS KS, VISCO AG. Mesh erosion in abdominal sacral colpopexy with and without concomitant hysterectomy. Am J Obstet Gynecol. 2006; 194:1418-22. [PubMed: 16647927]

22. NOSTI PA, LOWMAN JK, ZOLLINGER TW, HALE DS, WOODMAN PJ. Risk of mesh erosion after abdominal sacral colpoperineopexy with concomitant hysterectomy. Am J Obstet Gynecol. 2009; 201:541, e1-4. [PubMed: 19766973]

23. CUNDIFF GW, VARNER E, VISCO AG, et al. Risk factors for mesh/suture erosion following sacral colpopexy. Am J Obstet Gynecol. 2008; 199:688, e1-5. [PubMed: 18976976]

24. SIDDIQUI NY, GELLER EJ, VISCO AG. Symptomatic and anatomic 1-year outcomes after robotic and abdominal sacrocolpopexy. Am J Obstet Gynecol. 2012; 206:435, e1-5. [PubMed: 22397900]

25. OSMUNDSEN BC, CLARK A, GOLDSMITH C, et al. Mesh erosion in robotic sacrocolpopexy. Female Pelvic Med Reconstr Surg. 2012; 18:86-8. [PubMed: 22453317]

26. FRICK AC, WALTERS MD, LARKIN KS, BARBER MD. Risk of unanticipated abnormal gynecologic pathology at the time of hysterectomy for uterovaginal prolapse. Am J Obstet Gynecol. 2010; 202:507, e1-4. [PubMed: 20452498]

27. RAMM O, GLEASON JL, SEGAL S, ANTOSH DD, KENTON KS. Utility of preoperative endometrial assessment in asymptomatic women undergoing hysterectomy for pelvic floor dysfunction. Int Urogynecol J. 2012; 23:913-7. [PubMed: 22398824] 
28. BROWN JS, WAETJEN LE, SUBAK LL, THOM DH, VAN DEN EEDEN S, VITTINGHOFF E. Pelvic organ prolapse surgery in the United States, 1997. Am J Obstet Gynecol. 2002; 186:712-6. [PubMed: 11967496] 


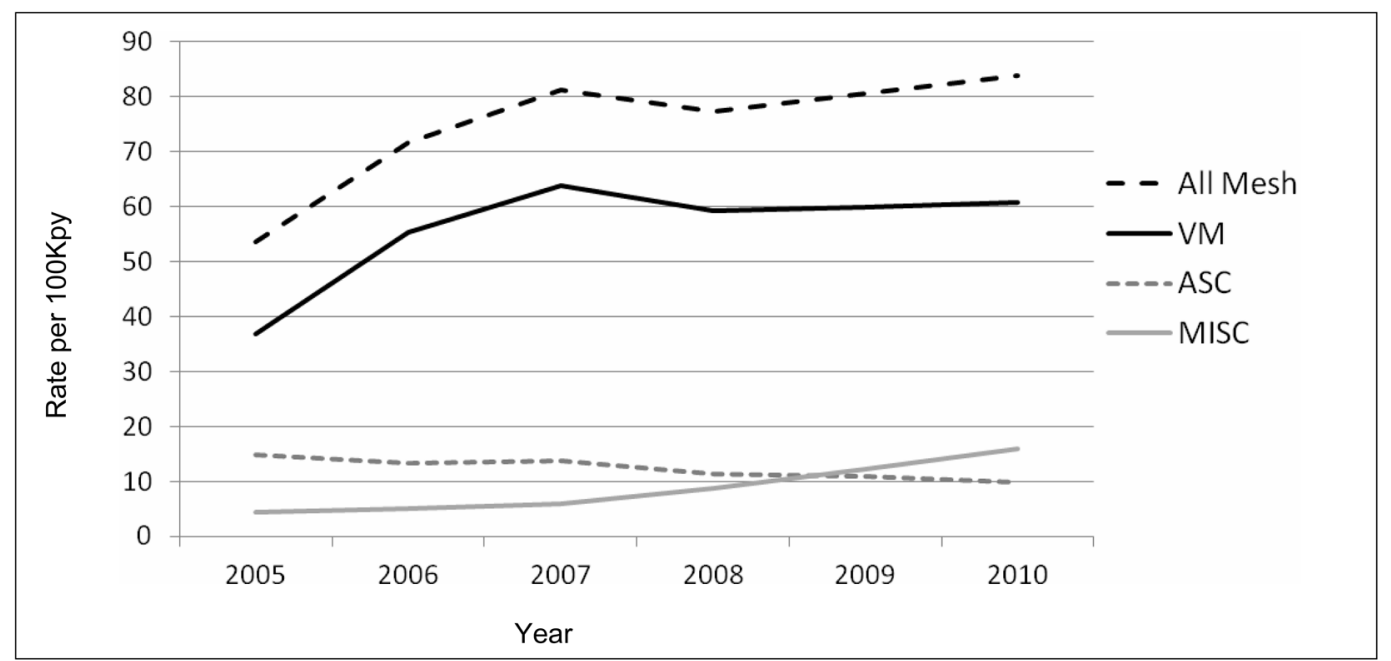

Figure 1. Annual rates of mesh prolapse procedures

Annual rates per 100,000 person-years for all mesh pelvic organ prolapse procedures, vaginal mesh (VM), abdominal sacrocolpopexy (ASC), minimally invasive sacrocolpopexy (MISC) from 2005-2010. Regarding the trend for the total number of prolapse mesh procedures (All Mesh) over calendar time, there was a significant increase $(\mathrm{p}<.0001)$. There was also a significant increase for VM procedures and MISC from 2005 to 2010 (p<.0001). In contrast, there was a significant decrease in the rate of ASC over calendar year $(\mathrm{p}<.0001)$. 


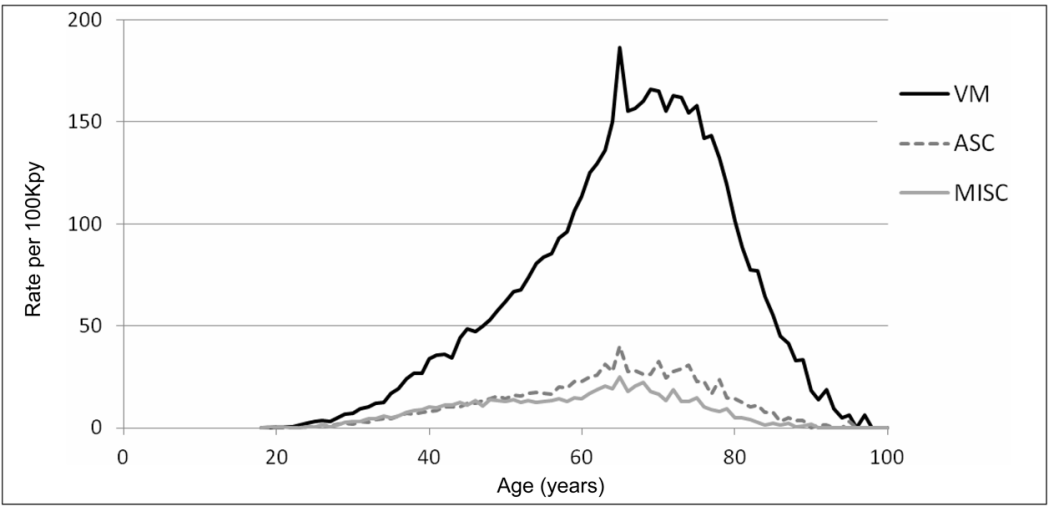

Figure 2. Age-specific rates for mesh prolapse procedures

Age-specific rates per 100,000 person-years for vaginal mesh (VM), abdominal sacrocolpopexy (ASC), minimally invasive sacrocolpopexy (MISC) performed from 20052010 , 


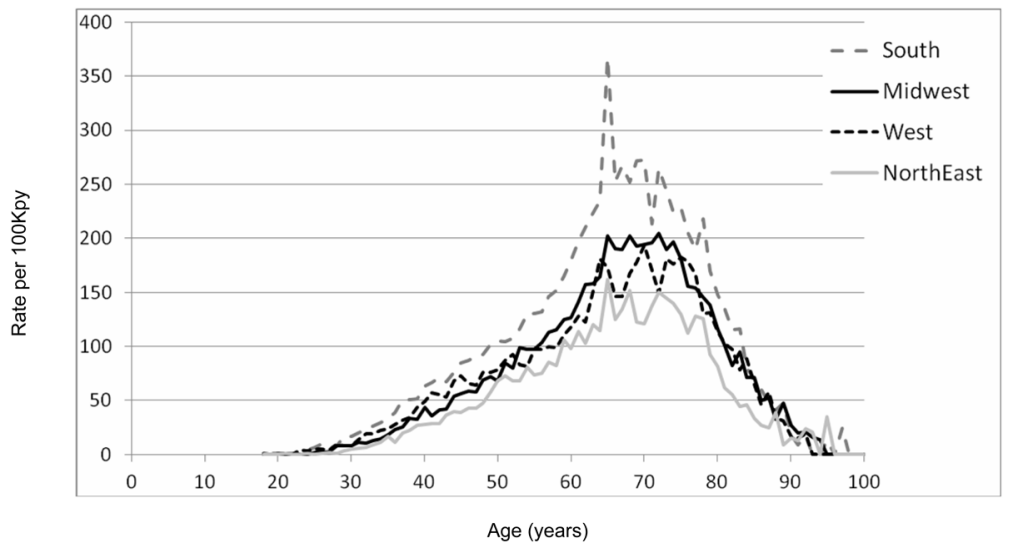

Figure 3. Age-specific rates for mesh prolapse procedures by region of the U.S Age-specific rates for prolapse mesh procedures performed from 2005-2010, stratified by region of the United States. When evaluating regional differences in prolapse mesh surgery rates after adjusting for age, all regions have significantly higher rates when compared to the Northeast $(\mathrm{p}<.0001)$. 
FUNK et al.

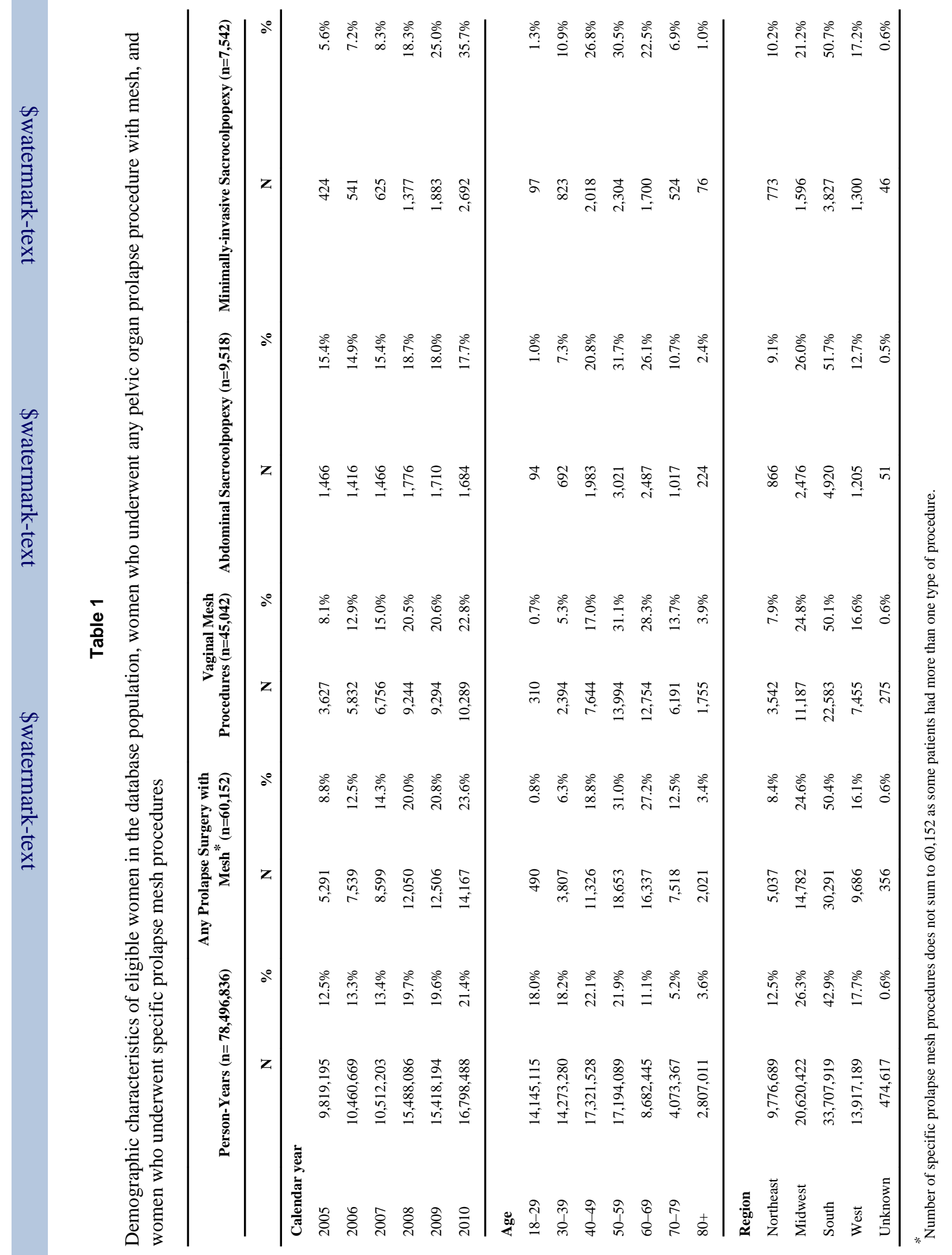

Am J Obstet Gynecol. Author manuscript; available in PMC 2014 January 01. 


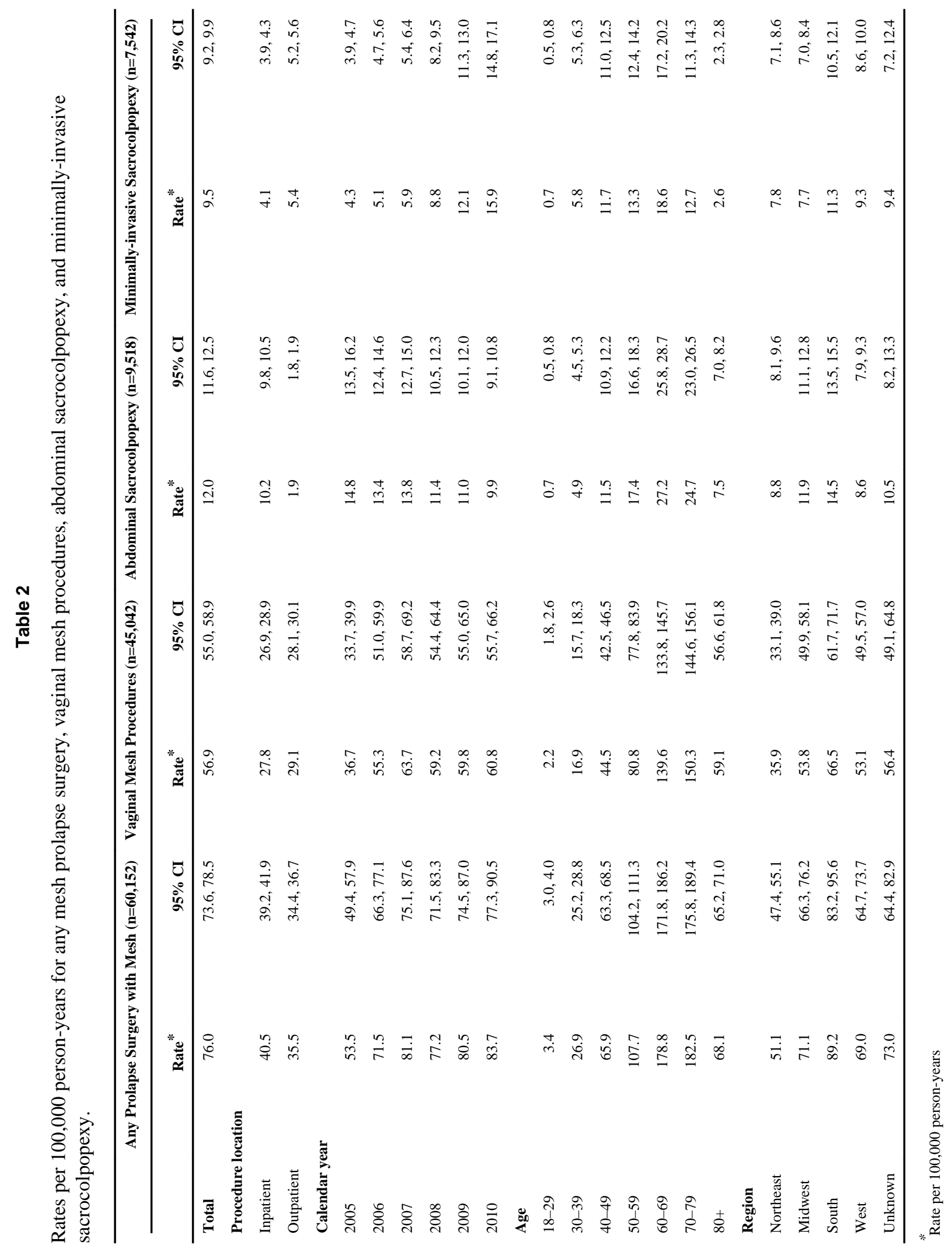

